\title{
THE CALCULUS IN OUR COLLEGES AND TECHNICAL SCHOOLS.
}

\author{
PRESIDENTIAL ADDRESS DELIVERED BEFORE THE AMERICAN \\ MATHEMATICAL SOOIETY APRIL 2\%, $190 \%$.
}

BY PROFESSOR WM. F. OSGOOD.

THE history of the race is frequently suggestive, in educational matters, of a wise course for the training of the individual. If we turn to the problems to which the calculus owes its origin, we find that not merely, not even primarily, geometry, but every other branch of mathematical physics - astronomy, mechanics, hydrodynamics, elasticity, gravitation, and later electricity and magnetism - in its fundamental concepts and basal laws contributed to its development and that the new science became the direct product of these influences.

Not until recent times have the analytic methods of the calculus been securely established. But at last the method of rates and that of infinitesimal constants have given place to the method of limits as being the only method known to us on which the calculus can be satisfactorily founded, satisfactorily, not merely from a logical point of view; it is important that the methods be in close touch with the physical concepts with which the calculus deals.

What is the bearing of these facts on instruction in the calculus in our colleges and technical schools? I wish to deal with some of the phases of this question.

\section{§ 1. Object of the Study of the Calculus.}

Let us first make clear to ourselves what some of the chief aims of the study of the calculus are. A view that has been widely accepted, to judge from the text-books that have had the largest sale, is that the first course should deal almost solely with the formal side. Just as the school boy learns to manipulate algebraic expressions with skill and accuracy, so the student shall first be drilled in differentiation and integration, the applications being chiefly to geometry and consisting in computing by formulas deduced in the text-book or class-room. 
Recently the pendulum has swung to the other extreme. The reformists have discovered that the engineer is well off if he can plot some simple curves from the tables and differentiate and integrate $x^{2}$ and $\sin x$, and we are told that the race has been degenerating under the old regime. Let us not lose perspective and let us not for a moment fail to recognize the fact that, whatever changes it may be desirable to make in the suggestive instruction of the course, the process by which the youth actually acquires the ideas of the calculus is to a large extent and essentially through formal work of substantial character. In order to attain this end, however, the formal work must appear to him as having for its direct object the power to solve some of the real problems of pure and applied mathematics, and these problems must always be kept before his eye.

Setting aside such secondary aims as the ability to recognize the derivatives and integrals that enter in the text-books of applied mathematics, let us ask ourselves what enduring service the study of the calculus can render to the man whose life work calls for a qualitative, and, perhaps, to some extent quantitative appreciation of physical phenomena. I believe one of the most valuable contributions of the calculus toward this end to lie in the training by which the student ultimately reaches the point of being able to discern and formulate those concepts and relations of physics that find their natural expression in definite integrals and differential equations. The road to the qualitative is through the quantitative. To be conscious that the flight of a golf ball is governed by Newton's laws of motion and to discern what the forces are that act and how they bring about the slice, is to have taken an important step toward dealing intelligently with dynamical questions in the construction of machinery or of a steamship. But the problem of the golf ball should not come till many, many simple quantitative problems in rectilinear and circular motion have been worked out by the student.

\section{§ 2. Differentials, Ancient and Modern.}

The original conception of the infinitesimal was that it is a very small, an "infinitely small," constant quantity, but not quite the absolute zero, a sort of little zero that was insignificant when compared with any of the quantities of every day life except zero, but that two of these differentials could be compared with each other in their microcosmos just as the quantities of 
every day life are comparable among themselves. Now this conception had its origin in a partial discernment of the truth, but, like the asymptotic series of astronomy, which converge only to a certain point and then diverge, it must not be pressed to the limit; for then the truth ceases and only chaos remains. Thus mathematicians have necessarily discarded the differentials of Leibniz as the elements out of which the calculus can be built up, and some are more than doubtful about the advisability of retaining them in any form.

We sometimes hear it said that hardly a theorem in our textbooks on the calculus is true as stated there. This is only a partial truth. Most of the theorems in the calculus are true when restricted as the authors intended they should be or as modern views readily suggest. And so here, in the case of the differentials, it behooves us to extract that which is true in the crude conception of former times and to shape a modern conception that is rigorous according to our present light.

There is a cogent reason for this course. The differentials are as firmly seated as ever they were in the methods of the physicists and engineers. We cannot banish them there, even if it were desirable to do so. Leave them out of the course in calculus and the student will meet them there with all the contradictions of the archaic infinitesimals, the little zeros of former times; and if he is capable of thinking clearly, he will see these contradictions and be helpless to reconcile them.

But beside this material reason there are two good scientific reasons for keeping the differentials and the infinitesimals. The former are useful in the formal work of differentiation and integration, the latter in the formulation of physical problems.

\section{§ 3. The Modern Conception of Infinitesimals.}

We have considered the archaic view of infinitesimals as small constants - a view still represented in the language, as when, for example, the President says: "The United States, owing to its peculiar position, has a regular army so small as to be infinitesimal when compared to that of any other first-class power." The modern scientific conception is that an infinitesimal is a variable which it is generally useful to consider only for values numerically small and which, when the formulation of the problem has progressed to a certain stage, is then allowed to approach zero as its limit. 
Two infinitesimals, $\alpha$ and $\beta$, are said to be of the same order if their ratio, $\beta / \alpha$, approaches a finite limit $K \neq 0$. If $K=0$, $\beta$ is said to be of higher order than $\alpha$; and if $K=\infty$, then $\beta$ is of lower order. If

$$
\lim \frac{\beta}{\alpha}=1, \quad \text { then } \quad \frac{\beta}{\alpha}=1+\zeta
$$

where $\zeta$ is infinitesimal, and hence $\alpha$ differs from $\beta$ by an infinitesimal $\zeta \alpha$ of higher order than $\alpha$ or $\beta$. Conversely, if $\alpha$ or $\beta$ differ from each other by an infinitesimal of higher order than that of either, then the limit of their ratio is unity. That infinitesimal which is chosen as independent variable is called the principal infinitesimal. If this be $\alpha$, then, since

$$
\lim \frac{\beta}{\alpha}=K, \quad \frac{\beta}{\alpha}=K+\zeta,
$$

and

$$
\beta=K a+\zeta a
$$

Thus $\beta$ differs from $K \alpha$ by an infinitesimal of higher order than $\alpha ; K a$ is called the principal part of $\beta$.

Two theorems lie at the foundation of the application of the calculus to natural philosophy.

Thforem 1. In the limit of the ratio of two infinitesimals, either infinitesimal may be replaced by one that differs from it by an infinitesimal of higher order :

$$
\lim \frac{\beta}{\alpha}=\lim \frac{\beta^{\prime}}{\alpha^{\prime}} \quad \text { if } \quad \lim \frac{\beta^{\prime}}{\beta^{\prime}}=1, \quad \lim \frac{\alpha^{\prime}}{\alpha}=1 .
$$

Theorem 2. Duhamel's Theorem. If a sum of infinitesimals, all of like sign, approaches a limit, then each of the infinitesimals may be replaced by one that differs from it by an infinitesimal of higher order :

$$
\begin{array}{cc} 
& \lim _{n=\infty}\left(\alpha_{1}+\alpha_{2}+\cdots+\alpha_{n}\right)=\lim _{n=\infty}\left(\beta_{1}+\beta_{2}+\cdots+\beta_{n}\right), \\
\text { if } & \lim _{n=\infty} \frac{\beta_{i}}{\alpha_{i}}=1 .
\end{array}
$$


§ 4. The Use of Infinitesimals in Formulating Physical Problems.

The importance of infinitesimals in the course in calculus which we are here considering lies in their use in formulating physical problems. A typical example or two will serve to make my meaning clear.

Let it be required to find the tension in a rope wound round a rough post and just on the point of slipping.

Consider a small arc $P P^{\prime}$ of the rope. We may regard it as a rigid body, since, if it became stiff, the forces would not necessarily thereby be disturbed. The forces that act on this

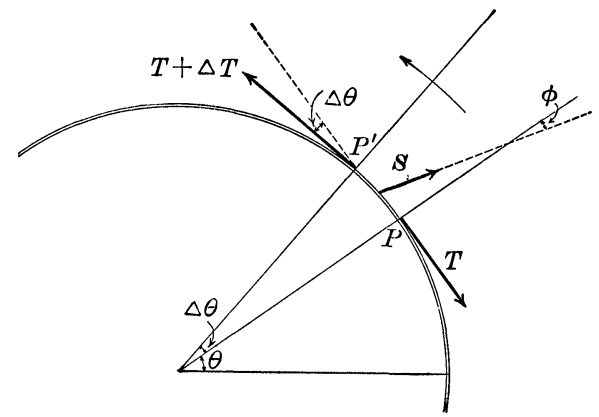

FIG. 1.

piece of the rope are : (1) the tension $T$ along the tangent at $P$; (2) the tension $T+\Delta T$ along the tangent at $P^{\prime}$; and (3) a resultant reaction $S$ of the cylinder; - the weight of the rope is unimportant in comparison with the forces considered. Resolving forces along the tangent and the normal at $P$, we get*

$$
\begin{aligned}
& (T+\Delta T) \cos \Delta \theta=S \sin \phi+T, \\
& (T+\Delta T) \sin \Delta \theta=S \cos \phi .
\end{aligned}
$$

Now if the rope is just on the point of slipping in the direction of the increasing angle $\theta$, friction will act in the direction of the decreasing angle, and thus $S$ will be inclined as shown in Fig. 1. What hypothesis shall we make about the direction of $S$ ? In other words, what is the physical fact? It is

* If the class is not familiar with the fact that, when a system of forces, acting on a rigid body, keep it at rest, they must be such a system as would be in equilibrium if they all acted at a single point, - then this theorem must, of course, be stated. 
easy to see if we think of a heavy flexible string $A B$ laid out straight on a rough horizontal table and pulled at one end $A$

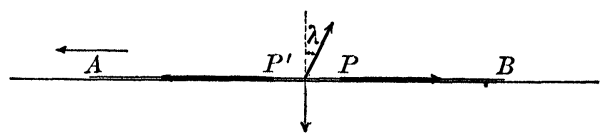

FIG. 2.

till it is just on the point of slipping. The total reaction $S$ of the table on a segment $P P^{\prime}$ here obviously makes an angle $\phi$ with the normal equal to the angle of friction $\lambda$.

In the case of the cylinder we have similar relations. The rope presses against the cylinder along the arc $P P^{\prime}$, which, being short, is but slightly curved, and hence the direction of $S$ will make an angle $\phi$ with the normal at $P$ which differs only slightly from the angle of friction $\lambda$, and approaches $\lambda$ as its limit when $P^{\prime}$ approaches $P$.

From equations (1) it follows that

$$
\begin{gathered}
\tan \phi=\frac{(T+\Delta T) \cos \Delta \theta-T}{(T+\Delta T) \sin \Delta \theta} \\
=\frac{\cos \Delta \theta}{T+\Delta T} \frac{\Delta T}{\sin \Delta \theta}-\frac{T}{T+\Delta T} \frac{1-\cos \Delta \theta}{\sin \Delta \theta} .
\end{gathered}
$$

We are now ready to allow $P^{\prime}$ to approach $P$ as its limit :

$$
\lim _{P P^{\prime}=0} \tan \phi=
$$$$
\left(\lim _{P P^{\prime} \doteq 0} \frac{\cos \Delta \theta}{T+\Delta T}\right)\left(\lim _{P P^{\prime} \doteq 0} \frac{\Delta T}{\sin \Delta \theta}\right)-\left(\operatorname{m}_{P P^{\prime} \doteq 0} \frac{T}{T+\Delta T}\right)\left(\lim _{P P^{\prime} \doteq 0} \frac{1-\cos \Delta \theta}{\sin \Delta \theta}\right) \text {. }
$$

The limit of $\tan \phi$ is $\mu$, the coefficient of friction. The limit of the first factor in each product is obvious, namely $1 / T$ and 1 respectively; for the tension increases gradually as we go along the rope, and so $\Delta T$ approaches 0 when $P^{\prime}$ approaches $P{ }^{*}$ In

* From equations ( 1 ) it can be proven that $\Delta T$ approaches 0 simultaneously with $\Delta \theta$; but the important view for the student at this stage is that the function ' $T$ changes continuously because it represents a physical quantity that, from its very nature, varies continuously. 
the limit of the second factor of the first term on the right, $\sin \Delta \theta$ can, by Theorem 1 of $\S 3$, be replaced by $\Delta \theta$ and hence

$$
\lim _{\Delta \theta \equiv 0} \frac{\Delta T}{\sin \Delta \theta}=\lim _{\Delta \theta=0} \frac{\Delta T}{\Delta \theta}=D_{\theta} T .
$$

The same replacement can be made in the last limit on the right; and since

$$
\lim _{a=0} \frac{1-\cos \alpha}{a}=0,
$$

the limit of the whole last term on the right is 0 . Our final result is, therefore,

$$
\mu=\frac{D_{\theta} T}{T},
$$

or

$$
\frac{d T}{T}=\mu d \theta .
$$

Hence, integrating, we get

and thus finally

$$
\log T=\mu \theta+C,
$$

$$
T=T_{0} e^{\mu \theta} .
$$

In the foregoing solution, which I have given in all detail, it will be observed that, in the formulation of the problem, the physical law is clearly stated : $\phi$ is approximately equal to $\lambda$ when $P^{\prime}$ is near $P$, and approaches $\lambda$ as its limit when $P^{\prime}$ approaches $P$. A difficulty which the student frequently meets in this border ground between mathematics and physics is that of finding out what the physical fact is. The physicist often shrinks from writing down a formula for fear that the student will not understand what it means, quite losing sight of the fact that it is just at this point that the student can most effectively be aided in forming clean cut physical conceptions, the natural expression of which is through mathematical symbols.

The physical law once granted, equations (1) are absolutely true, whether the arc $P P^{\prime}$ subtends an angle of $10^{\circ}$ or $.000001^{\prime \prime}$. Moreover, in taking the limits, nothing is " neglected." And the limits lead us directly to derivatives, not to differentials. The 
differentials enter only subsequently through the derivatives. If the differentials are introduced at the outset, $\theta$ being taken as the independent variable, the force at $P^{\prime}$, namely $T+\Delta T$, is not $T+d T$; it differs from the latter quantity by an infinitesimal of higher order. And the equations (1), written with the $\Delta$ 's replaced by the $d$ 's and $\phi$ by $\lambda$, are not true till a correction in the form of an infinitesimal of higher order has been added to one side of each equation. Thus the use of differentials in formulating the problem, if carried out rigorously, only detracts from the simplicity of the equations and at best replaces the quantities that naturally enter by artificial ones not quite equal to them. In the formulation of physical problems leading to definite integrals - areas and volumes, centres of gravity, moments of inertia, fluid pressures, attraction of gravitation, work and energy, kinetic energy, etc. - the situation is similar. First, the quantity to be computed is divided into $n$ infinitesimal parts, $i$. $e_{\text {. }}$, into $n$ definite pieces, the magnitude of each of which is expressed accurately by an analytic symbol, and the quantity in question is precisely the sum of these pieces. Hence it is also equal to the limit of their sum. Then the $i$ th part is approximated to by means of an infinitesimal which, in the case of a simple integral, is of the form $f\left(x_{i}\right) \Delta x_{i}$ and which differs from the $i$ th infinitesimal in the above sum by an infinitesimal of higher order. By virtue of Duhamel's theorem the limit of the second sum,

$$
\lim _{n=\infty} \sum_{i=1}^{n} f\left(x_{i}\right) \Delta x_{i},
$$

is equal to that of the first sum, and thus the quantity to be computed is formulated as the definite integral

$$
\int_{a}^{b} f(x) d x .
$$

Here, again, nothing is "neglected." There is a clean cut formulation of the quantity in question as a sum, hence as the limit of that sum, and then follows the evaluation of this limit by means of a definite integral.

These are two of the central problems of the calculus, and an intelligent solution of them depends on a clear and adequate 
understanding of what infinitesimals are and how to use them. No greater mistake can be made than to think that these problems belong in the applications of the calculus in the courses in physics and engineering. This view is a wrong one for two reasons. First, these problems are essential for understanding what the calculus is. Poincare has said that it is nature which forced the continuum on mathematicians. If they had been left the prey of abstract logic, they would never have gotten beyond the theory of numbers and the postulates of geometry. Now, to understand the continuum, we must begin with continuous variation and the conception of the function. In no other way are these ideas brought home to our feeling, our intuition, as through the concepts and the simple laws of natural philosophy, beginning with the geometric problems of mensuration. To think that there is one calculus for the pure mathematician and another for the physicist, the engineer, the geometer, or the cultured layman, is to fail to appreciate that that which is most central in the calculus is its quantitative character, through which it measures and estimates the things of the world of our senses. And instruction in the calculus that does not point out - not merely at the beginning or at the end, but all through the course - this close contact with nature, has not done its duty by the student.

Thus we need these problems of natural philosophy in the course in calculus primarily for their own sake, alike for the training of the men who are to go on with mathematics and of those who are not. But there is another reason why we should treat these problems in the course in the calculus, and that is that the teachers in the courses in applied mathematics are frequently ignorant of modern methods in the calculus. Infinitesimals were given to them as small constants, zero, but yet not zero, that can be kept or thrown away at the pleasure of the instructor. They accepted the situation, and if their scientific consciences ever protested, they have long since been silenced. As I said at the beginning, there is a partial truth contained in this archaic view of the infinitesimal, and this is enough for some students. But our colleagues in applied mathematics make a huge mistake when they assume, as mathematicians once did, that their little zeros satisfy their best students. I remember hearing my father relate an amusing anecdote from his college days, which shows how abhorrent this juggling with infinites is to a healthy mind still able to 
reject the poison. It was in the class in geometry and Professor P. had called on Mr. B. to prove the theorem by which the area of a circle is expressed in terms of the length of its circumference. Mr. B. had learned his lesson from the professor's text-book, in which it was set forth that a circle is a polygon of an infinite number of sides; and he recited the proof down to the conclusion, and there he stopped. "What does that prove?" asked the professor. "It proves an absurdity!" was the reply.

The science has advanced since then. We no longer have the infinite and the infinitesimal constants of former days in mathematics. If we are going to replace them in the courses in applied mathematics by the true infinites and infinitesimals, the variables, we must begin by teaching in the courses in mathematics what these variables are and how to use them.

\section{§ 5. The Place of the Differential.}

The course in calculus opens with finding the slopes of curves and the derivatives of the elementary functions, and makes application to maxima and minima and to some extent, too, to curve plotting; the more substantial problems in velocities coming a bit later. Here, the limit of the ratio, $\Delta y / \Delta x$, is the central idea, the key to the situation, and the attention of the student should not be embarrassed by any attempt to introduce infinitesimals at this first stage. $\Delta y / \Delta x$ is a function of the independent variable $\Delta x$ and approaches, in all cases considered, a definite limit (or possibly, for certain values of $x_{0}$, becomes infinite) when $\Delta x$ approaches 0 . Its limit should be denoted by an expression that does not suggest a ratio. The expressions of this sort in common use are

$$
D_{x} y, \quad y^{\prime} \text { or } y_{x}, f^{\prime}(x) .
$$

But the last three are too meagre a notation for the beginner, and so one naturally chooses $D_{x} y$.

Practical considerations make it necessary to introduce the differential at an early stage. The student meets the differential notation in his courses in applied mathematics and so it must be explained early in the course in the calculus. My experience, which I notice is confirmed in substance by that of recent writers, leads me to bring in the differential as soon as 
the differentiation of the algebraic functions has been completed and the applications above mentioned taken up, but while there is still enough drill work in formal differentiation left so that the student will become thoroughly familiar with the method. Since we must teach differentials before the student is ready for them, we may at least get the advantage in technique which accrues from them in such differentiations as

$$
d \sqrt{a^{2}-x^{2}}=\frac{1}{2} \frac{1}{\sqrt{a^{2}-x^{2}}} d\left(a^{2}-x^{2}\right)=\text { etc. }
$$

The differential is to be introduced as an infinitesimal, a variable, coordinate with the increments $\Delta x, \Delta y$, etc., and differing from its corresponding increment by an infinitesimal of higher order. $d y$ is the principal part of $\Delta y, \Delta x$ being taken as the principal infinitesimal. This is the first, but by no means the last word to be said in the course about infinitesimals, and the teacher must make this preliminary exposition brief, but incisive, a careful explanation of the usual geometric figure for illustrating $\Delta x=d x, \Delta y$, and $d y$ being sufficient for the purpose.

In practice the important side of differentials is the formal side. They form one of the oldest examples of homogeneous variables and their chief value in the calculus consists in their application in formal differentiations and integrations. They appear to good advantage, too, in problems in velocities, for by their aid these problems can be reduced to solving some homogeneous equations in $d s, d x, d y, \cdots, d t$ for $d s$ in terms of $d t$.

\section{$\S 6$. The Service of Modern Analysis to the Calculus.}

A question that many of us have asked ourselves is: What service has modern progress in analysis rendered to the calculus of the undergraduate curriculum? At one time it looked as if recent advances in analysis were but to veil the conceptions of the calculus in a mist of $\epsilon$ 's. But maturer thought disclosed that the formal $\epsilon, \delta$-proof is not needed till questions in double limits are reached, and the treatment of these questions belongs to a later stage. The fundamental idea of the limit is that a variable point, restricted according to the conditions of the problem under consideration, ultimately comes and remains within any arbitrarily assigned neighborhood of a certain fixed point, no matter how small this neighborhood may be chosen. 
In the development of analysis during the nineteenth century this conception had to meet two demands. The first was that of its adaptability to the needs of the growing science; the second was the refinement which mathematics requires of the ideas which it accepts as fundamental. The fact that it stood both of these tests satisfactorily was an important factor in determining mathematicians to base the calculus on limits, and not on the infinitesimals of Leibniz or the rates of Newton.

A further service of modern analysis lies in its recognition of the elementary theorems about continuity as a secure foundation, a reliable method. If a function $f(x)$, continuous in the interval $a \leqq x \leqq b$, vanishes at the extremities of the interval and is positive at all interior points, or if, more generally, it has at some interior point a value larger than either $f(a)$ or $f(b)$, then it has a maximum value, and in fact a maximum maximorum, within the interval. Likewise, if $f(x)$ becomes positively infinite at each extremity of the above interval and is continuous at all intermediate points, it has a minimum value within the interval. These principles can be used with advantage in the problems in maxima and minima at the beginning of the course. It is important that the student should plot roughly the graphs of the functions he tests for maxima and minima, partly that he may avoid the error of mistaking a relative maximum or minimum at an interior point for a minimum minimorum at an extremity of the interval,* but more especially to give him practice in thinking of a function geometrically as a curve and in reasoning about its properties from inspection of the curve. Nearly all the cases that arise in practice, the "clothed problems," can be dealt with rigorously in this manner without the use of the second derivative, the reasoning being (1) that there is at least one maximum or minimum within the interval ; (2) that the first derivative of the function (since it exists at each point within the interval) must vanish there; and then it turns out (3) that the latter equation has only one root in the interval.

Thus the second derivative is not usually needed for the problems of maxima and minima that arise in practice. It is, however, of value to have an analytic test for relative maxima

* Professor Huntington has given a neat example of this case. A bather standing on the bank of a circular pond, wishes to reach the point directly opposite in the shortest possible time. If he can swim at the rate of $u$ miles an hour and run at the rate of $v$ miles an hour, what point should he swim toward? 
and minima. Now the most instructive form in which this test can be presented at this early stage in the course is as a problem in curve plotting, and this leads me to say a few words about the right and the wrong way to plot curves.

We need a few graphs accurately plotted because of the importance of the functions they represent; namely, the graphs of $\sin x, \cos x, \tan x, \log x$ and the inverse functions, and $x^{n}$, $n>0, x \geqq 0$. These the student can plot directly from the tables (but he won't do it correctly unless the instructor has good staying powers). What we usually want to know about the graph of a function in the calculus is its character, and even when we need quantitative accuracy, a knowledge of the character of the curve at the outset is a help in plotting. Now the calculus affords a powerful aid for determining the character of a function. If $y=f(x)$ and $D_{x} y$ is positive, $y$ is increasing with $x$; if negative, decreasing. Furthermore, if $D_{x} \tan \tau=D_{x}^{2} y$ is positive, the slope of the curve is increasing with $x$ and so the curve is concave upward; if $D_{x}^{2} y$ is negative, the curve is concave downward. In addition to these principles we have the elementary theorems of modern analysis about continuous functions.

That my meaning may be perfectly clear at this point, consider the familiar example :

Here

$$
y=x^{3}+p x+q .
$$

$$
D_{x} y=3 x^{2}+p, \quad D_{x}^{2} y=6 x .
$$

From the last equation we see that for all negative values of $x$ the curve is concave downward, for positive values concave upward. If $q=0$, the curve is symmetric with respect to the origin; otherwise with respect to the point $x=0, y=q$. Finally, since

$$
\left.y\right|_{x=-\infty}=-\infty,
$$

we have the character of the curve in its entirety without computing the coordinates of a single point except those of the point of inflection. The slope at that point is $p$ and thus if $p \geqq 0$, the cubic equation

$$
x^{3}+p x+q=0
$$


has but one real root. If $p<0$, the coordinates of the two maxima and minima tell the story. Other examples of this kind can serve as exercises and thus the graphs of polynomials and rational functions, if treated by the methods of the calculus above mentioned, can be used to advantage for training in the calculus.

It is a mistake, however, to dwell very long on these exercises near the beginning of the course. The student learns to apply the above principles by getting examples of their use when he is not looking for them. In one clothed problem or another he will come upon such an equation as

$$
\theta-\tan \theta=0 \text {. }
$$

One root is $\theta=0$. Is there any further root in the interval from $\theta=0$ to $\theta=\pi / 2$ ? Let him work out this problem without any suggestions, or only with meagre ones. And when he has finally seen the point, let him find out how many roots the equation has in the interval from $\theta=\pi / 2$ to $\theta=3 \pi / 2$. Other equations of similar type are :

$$
\begin{gathered}
a \theta=\tan \theta \quad(a \neq 1), \\
\theta(1+\cos \theta)-2 \sin \theta=0, \\
\left(1+b^{2}\right) \tan ^{-1} b=b .
\end{gathered}
$$

And when the orthogonal families of confocal ellipses and hyperbolas are taken up, let him show analytically that the equation in $\lambda$ :

$$
\frac{x^{2}}{a^{2}+\lambda}+\frac{y^{2}}{b^{2}+\lambda}=1,
$$

has one and only one root in each of the intervals $-a^{2}<\lambda<-b^{2}$ and $-b^{2}<\lambda<+\infty$.

A simple problem in maxima and minima that calls for curve tracing as here set forth is the following: A gutter whose cross-section is an arc of a circle is to be made by bending into shape a strip of copper. If the width of the strip is $a$, find the radius of the cross-section when the carrying capacity of the gutter is a maximum. 
In the second course in the calculus the $\Gamma$-function is introduced by the integral

$$
\Gamma(\alpha)=\int_{0} x^{\alpha-1} e^{-x} d x \quad(\alpha>0),
$$

and its derivatives are given by differentiating under the sign of integration. Assuming this process to be justifiable, let the class determine the character of the graph of the function.

Here, then, is another great principle of the calculus. It is not quickly learned. To inculcate it into the student, it is not necessary, however, nor even desirable, to take any large amount of time from other things. What is required is vigilance on the part of the teacher. The psychological moment will not come with all students, or even many, in a given hour. It is necessary to give the class successive opportunities, spread out over the whole course, so that the environment may frequently be favorable for the reception of the desired suggestion. But when a student has once come to incorporate this principle into his mode of thought, he has received from the calculus an aid to that of which I spoke at the beginning - the power to grasp, through the quantitative, the qualitative side of the physical world about us.

\section{§ 7. Analytic Mechanics.}

A further subject of importance for the training of the undergraduate in mathematics is that of mechanics. This subject is treated most satisfactorily, I believe, in a collateral course in charge of the department of mathematics, provided that the instructor in this course has clear insight into the elementary facts of mechanics and their exemplification in every day life, and is interested primarily in teaching mechanics, not analytic geometry and the calculus. It is desirable to begin with the statics of particles and rigid bodies, treated both analytically and graphically. In the statics of a particle the force polygon, drawn to scale, serves as a check on the analytic solution. Half a dozen lectures on the graphical statics of roof and bridge trusses, including the graphical determination of centres of gravity, prove highly interesting to a college class. Thus the student has been clarifying his ideas of force before the dynamics of moving bodies is begun, and in the meantime he has learned in the course in the calculus how to differentiate and inte- 
grate. He is now ready for Newton's second law of motion, a desirable foundation for dynamics for the beginner, since it makes him think about inertia and the way a body moves under the action of forces, as well as about the reaction of the parts of a machine on their constraints. The course naturally includes an extended treatment of centrifugal force, the principle of work and energy, and the dynamics of a rigid body in the case of motion in two dimensions. It is also important to set forth the conceptions and the fundamental theorems about the instantaneous center, the space and the body centrode, and the law of cam motion.

From the time dynamics is begun, - about one third of the course should be given to statics, - the calculus is of vital importance. On the other hand the problems, several of which should be worked independently by the student in connection with each lecture, ought not, as a rule, to involve serious difficulties of an analytic nature. There ought to be many easy and some hard exercises; but the difficulty of the latter should be due to the incompleteness of the students mechanical intuition, not to complicated analytic or geometric considerations.*

The course in the calculus ought to contain a chapter in mechanics, in which rectilinear motion under forces that are functions of the space or of the velocity are treated and which thus supplements the course in mechanics without duplicating it. If, however, such a course in mechanics as the above is not given, this chapter must be lengthened; but it cannot in any case replace the course in mechanics. I have for many years given both courses, and I have found the course in mechanics to afford most valuable training in the appreciation of some of the leading principles of the calculus; and this in spite - or perhaps rather because - of the fact that I reduce the analy tic difficulties of the course to a minimum.

\footnotetext{
* In technical schools the course in graphical statics, if prescribed for all who take this course, makes it possible to abridge slightly the treatment of statics. But the course we are here considering is otherwise independent of that course and the course on the strength of materials.

It is, of course, desirable that a second course in mechanics be given. But inasmuch as a first course of the elementary character here outlined is frequently lacking and is for most students the more instructive of the two, it is a far more diffioult course to give effectively than the advanced course is, - I am concerned here with emphasizing this gap in mathematical instruction.
} 


\section{§ 8. Advanced Calculus.}

The first course in the calculus, even though it be a five hour course preceded by differentiation in the freshman year, is not adequate for covering all the topics that belong in a proper treatment of the elementary part of the subject. Take, for example, partial differentiation. The easier applications to problems in the geometry of space can be treated in the first course. But the more difficult applications which arise in pure mathematics and in physics, and which are largely formal, clearly should find a place in the second course. Again, in multiple integrals, the conception of the multiple integral, its evaluation by means of iterated integrals, and its use in formulating physical problems belong early, and in technical schools the instruction should be so arranged that all students of engineering will get this part of the subject. But Green's theorem and its applications to exact differentials and to such problems as the deduction of Laplace's equation as the condition for a steady flow of heat or electricity claim an important place in the second course.

Then there are the hyperbolic functions, the elliptic integrals and functions, and the $\Gamma$ - and B-functions, the latter being defined by definite integrals. When I say that the course should treat thoroughly the simplest tests for the convergence of improper definite integrals, you will all agree with me and some will wonder why I do not demand more, tests for continuity, etc. The reason is that the student is not ready to receive more at this stage. If those of you who give a second course in the calculus will inform yourselves about the student's real power in dealing with the simplest cases where he has to detect unaided whether the integral in question is a proper or an improper integral, you will find a degree of immaturity hard to realize. He will test, for example, the integral

$$
\int_{0}^{1} \frac{\log x}{1-x} d x
$$

for convergence or divergence at the upper end of the interval of integration with the same care that he bestows on the lower end. "Have him plot the graph of the integrand," you say. Good advice; this is an aid. But ask the class to note the time they put on the exercises that are handed in, and take the lesson to heart. The fact is that the student is nowhere near 
the point where he can even see at a glance in all the simpler cases whether the integrand becomes infinite or remains finite; much less is it easily apparent to him whether to expect convergence or divergence, and what test to apply. Now these are the very things that have to be taught him right here and it takes time. But it is time well spent; it is the teacher's opportunity to strengthen the student's power to deal with functions and at the same time to treat the formal side of the definition of functions by means of definite integrals, the only questions of convergence here considered being those of the mere convergence of the integrals that present themselves. How far beside the mark double limits and $\epsilon$-proofs are at this stage is clear to every teacher who has perceived his opportunity at this point.

The value of a special course on elementary differential equations is generally recognized. But, wholly aside from this course, differential equations should permeate both courses in the calculus. Through them clothed problems of first importance for training in the calculus are introduced.

I have not even mentioned the subjects of the further elementary developments of infinite series, including Fourier's series, of mean value and probability, of the approximate solution of equations, and of a brief introduction to the theory of functions of a complex variable and the calculus of variations. And yet most of these subjects should be studied in an elementary way before the more difficult questions of modern analysis are begun.

This, then, is the elementary material that forms the subject matter of the calculus. To introduce the treatment of questions in double limits at this stage is both to deprive the student of the chance to acquire a broad knowledge of the calculus and to bungle his training in analysis.

\section{§9. Conclusion.}

It has not been my intention to mark out a course in the calculus, for the great body of the instruction at present given by any good teacher is sound and ought to be retained in substance. Before all, there is the necessity of faithful insistence on the part of the instructor on accurate formal work of reasonably substantial character. The technique of the calculus must be taught. Then there is the large body of easy problems in 
the applications which depart so slightly from the typical problems of the text-books or the class-room that they are within the reach of all who make an honest effort to do the work of the course. Moreover, as regards the choice of material, the important improvement of introducing the integral as the limit of a sum early in the first course may now be fairly regarded as achieved. And yet, with all of this that is so good and sound, if you open one of our text-books on the calculus and ask: What is the calculus? What will abide after the formulas are forgotten? What is the soul and the spirit of this great science, as conceived by the man whose work in life does not lie within the field of mathematics? I can't help feeling that the answer does not ring clear: The calculus is the greatest aid we have to the appreciation of physical truth in the broadest sense of the word.

\section{NOTES.}

The fourteenth summer meeting of the American Mathematical Societr will he held at Cornell University on Thursday-Friday, September 5-6. Titles and abstracts of papers to be presented at this meeting should be in the hands of the Secretary by August 24. Abstracts intended to be printed in the announcement of the meeting must be submitted by August 17.

The April number (volume 8, number 2) of the Transactions of the American Mathematical Society contains the following papers: "Dynamical trajectories: the motion of a particle in an arbitrary field of force," by E. KASNER ; "A class of periodic orbits of an infinitesimal body subject to the attraction of $n$ finite bodies," by W. R. LONGLEY ; "A proof of some theorems on pointwise discontinuous functions," by E. B. VAN VILCK ; "Invariants of binary forms under modular transformations," by L. E. Dickson ; "Projective differential geometry of curved surfaces (first memoir)," by E. J. WILCZYNSKI; "A method for constructing the fundamental region of a discontinuous group of transformations," by J. I. HuTcHINSON ; "Oblique reflections and unimodular strains," by E. B. WILSON ; "On the introduction of convergence factors into summable series and summable integrals," by C. N. Moore. 